\title{
Coupled intervals for discrete $p$-degree functional
}

Robert Mař́k ${ }^{*}$

${ }^{\text {*Correspondence: }}$
marik@mendelu.cz
Department of Mathematics,
Mendel University in Brno,
Zemědělská 3, Brno, 61300, Czech
Republic

\section{Abstract}

We study the scalar $p$-degree functional

$$
J(x)=R_{N+1}\left|x_{N+1}\right|^{p}+\sum_{k=0}^{N}\left[R_{k}\left|\Delta x_{k}\right|^{p}-C_{k}\left|x_{k+1}\right|^{p}\right]
$$

over the class of sequences with zero boundary condition at the left endpoint and free right endpoint. We extend the linear concept of coupled intervals to give a necessary and sufficient condition for nonnegativity and positivity of this functional.

MSC: 39A12; 49K99

Keywords: half-linear difference equation; $p$-degree functional; coupled interval; conjugate point

\section{Introduction}

In this article, we study the $p$-degree functional

$$
J(x)=R_{N+1}\left|x_{N+1}\right|^{p}+\sum_{k=0}^{N}\left[R_{k}\left|\Delta x_{k}\right|^{p}-C_{k}\left|x_{k+1}\right|^{p}\right],
$$

where $N \geq 1, R_{k} \neq 0$ for $k \in[0, N]$ and $p>1$. (Note that as usual, under the interval we actually mean the intersection of the interval of real numbers with the set of all integers.) The domain of the functional $J$ (the class of admissible functions) is supposed to be the set of functions $x$ defined on $[0, N+1]$ which satisfy $x_{0}=0$. The functional is said to be nonnegative over the class of admissible functions if $J(x) \geq 0$ for every admissible $x$. Similarly, $J$ is said to be positive if $J(x)>0$ for every admissible $x$ which is not equal zero identically on $[0, N+1]$.

This article can be considered as a continuation of the article [1] dealing with the $p$ degree functional (1) over the class of the functions satisfying $x_{0}=0=x_{N+1}$ and the article [2], where the functional

$$
A\left|x_{0}\right|^{p}+\sum_{k=0}^{N}\left[R_{k}\left|\Delta x_{k}\right|^{p}-C_{k}\left|x_{k+1}\right|^{p}\right],
$$

defined over the class of nontrivial sequences satisfying $x_{N+1}=0$ has been studied. It turns out that the concept of conjugate intervals which has been used in $[2,3]$ is no more suf-

\section{Springer}

(c) 2012 Mař́k; licensee Springer. This is an Open Access article distributed under the terms of the Creative Commons Attribution License (http://creativecommons.org/licenses/by/2.0), which permits unrestricted use, distribution, and reproduction in any medium, provided the original work is properly cited. 
ficient to prove nonnegativity and/or positivity of the functional (1). For this reason, we use a different concept in this article - the concept of coupled intervals developed for the quadratic functional

$$
\mathcal{I}=\left(\begin{array}{c}
\eta_{0} \\
\eta_{N+1}
\end{array}\right)^{T} \Gamma\left(\begin{array}{c}
\eta_{0} \\
\eta_{N+1}
\end{array}\right)+\sum_{k=0}^{N}\left\{\eta_{k+1}^{T} P_{k} \eta_{k+1}+2 \eta_{k+1}^{T} Q_{k} \Delta \eta_{k}+\Delta \eta_{k}^{T} R_{k} \Delta \eta_{k}\right\}
$$

in a series of papers written by Šimon Hilscher and Zeidan, see [4-6] and the references therein. Note that the theory of coupled points for differential equations has been developed in a series of articles written by Došlá, Došlý, Zeidan and Zezza, see [7-10] and the references therein. The extension of the continuous coupled points theory from the quadratic to the $p$-degree functional is described in [11].

The quadratic functional (2) defined over various classes of admissible functions is studied extensively in the literature because of the connection with the second variation: (2) is a second variation of the functional

$$
K\left(x_{0}, x_{N+1}\right)+\sum_{k=0}^{N} g\left(k, x_{k+1}, \Delta x_{k}\right)
$$

see [4] for details. Note that in contrast to [4], we deal with the $p$-degree functional and for this reason we work with the scalar case only. In this case, the matrix products in (2) reduce to the products and powers of real numbers.

The corresponding Euler-Lagrange equation to the functional (1) is the half-linear difference equation

$$
\Delta\left(R_{k} \Phi\left(\Delta z_{k}\right)\right)+C_{k} \Phi\left(z_{k+1}\right)=0
$$

where $\Phi(t)=|t|^{p-2} t$. Equation (3) has the property that a constant multiple of every solution is also a solution of this equation. See also [3, Chapter 8] for basic results concerning equation (3). If $p=2$, then the $p$-degree functional reduces to the quadratic functional and the half-linear equation (3) reduces to the linear equation.

There are many similarities between equation (3) and the linear second-order differential or difference equations. Among others, many results from the comparison and oscillation theory of the linear second-order difference equation extend to (3). Recall that in the oscillation theory of the second-order difference equations, we replace the zeros of continuous functions by the so-called generalized zeros, defined as follows:

Definition 1.1 (Generalized zero) Let $z$ be a solution of (3). The interval $(m, m+1]$ is said to be a generalized zero of the solution $z$ if $z_{m} \neq 0$ and $R_{m} z_{m} z_{m+1} \leq 0$.

Definition 1.2 (Conjugate interval) The interval $(m, m+1]$ is said to be conjugate to 0 if $(m, m+1]$ is a generalized zero of the solution $z$ which satisfy $z_{0}=0, r_{0} \Phi\left(z_{1}\right)=1$.

The functional (1) defined over the class of functions satisfying $x_{0}=0=x_{N+1}$ is known to be positive definite if and only if there is no conjugate interval to the point 0 (i.e. there is no generalized zero of the solution which satisfies $x_{0}=0, r_{0} \Phi\left(x_{1}\right)=1$ ) on the interval $(0, N+1]$, see [3, Theorem 8.2.2]. Since the class of sequences with the zero boundary 
conditions on both $x_{0}$ and $x_{N+1}$ is a subset of the class of sequences with zero boundary condition at 0 , the nonnegativity (positivity) of the functional (1) over the class of functions which satisfy $x_{0}=0$ implies the nonnegativity (positivity) of this functional over the class of functions which satisfy $x_{0}=0=x_{N+1}$. Hence, the positivity of the functional (1) also implies the nonexistence of the interval conjugate to the point 0 on $(0, N+1]$.

The following definition is motivated by [6]. For a deeper insight and a motivation for this definition, see also Remark 1.1.

Definition 1.3 (Coupled interval) Consider functional (1) and let $m \leq N$. The interval $(m, m+1]$ is said to be coupled with 0 if there exists a solution $z$ of $(3)$ on $[0, m-1]$ and a real number $\alpha$ which satisfy $z_{0}=0, r_{0} \Phi\left(z_{1}\right)=1, z_{m} \neq 0$,

$$
d_{m}:=R_{m} z_{m} \Phi\left(\Delta z_{m}\right)+R_{m}\left|\alpha-z_{m}\right|^{p}+|\alpha|^{p}\left(R_{N+1}-\sum_{k=m}^{N} C_{k}\right) \leq 0
$$

and if $m \neq N$ then also $z \not \equiv \alpha$ on $[m+1, N+1]$. If the strict inequality $d_{m}<0$ holds, then $(m, m+1]$ is said to be strictly coupled with 0 .

Remark 1.1 Note that from the condition $z_{0}=0 \neq z_{m}$, it follows that $(0,1]$ cannot be coupled with 0 . The quantity $d_{m}$ from (4) is closely related to the value of the functional along an admissible function which follows the solution starting with zero value and which is extended as a constant sequence from some index. To show this connection let $z$ be a nontrivial solution of (3) on $[0, N-1]$ such that $z_{0}=0, R_{0} \Phi\left(z_{1}\right)=1$ and for $m \leq N$ consider the sequence $x$ defined by

$$
x_{k}= \begin{cases}z_{k}, & k \in[0, m], \\ \alpha, & k \in[m+1, N+1],\end{cases}
$$

i.e. $x$ is a solution on $[0, m-2], \Delta x_{m}=\alpha-z_{m}$ and $\Delta x_{k}=0$ for $k \in[m+1, N]$. Now a direct computation shows

$$
J(x)=R_{N+1}|\alpha|^{p}+\sum_{k=0}^{m-1}\left(R_{k}\left|\Delta z_{k}\right|^{p}-C_{k}\left|z_{k+1}\right|^{p}\right)+R_{m}\left|\alpha-z_{m}\right|^{p}-\sum_{k=m}^{N} C_{k}|\alpha|^{p} .
$$

Using summation by parts, $z_{0}=0$ and in view of the fact that $z$ is a solution on $[0, m-1]$ we have

$$
\begin{aligned}
& \sum_{k=0}^{m-1}\left(R_{k}\left|\Delta z_{k}\right|^{p}-C_{k}\left|z_{k+1}\right|^{p}\right) \\
& \quad=R_{m} z_{m} \Phi\left(\Delta z_{m}\right)-R_{0} z_{0} \Phi\left(\Delta z_{0}\right)-\sum_{k=0}^{m-1}\left(\Delta\left(R_{k} \Phi\left(\Delta z_{k}\right)\right)+C_{k} \Phi\left(z_{k+1}\right)\right) z_{k+1} \\
& \quad=R_{m} z_{m} \Phi\left(\Delta z_{m}\right)
\end{aligned}
$$

and hence $J(x)=d_{m}$. 
Remark 1.2 If not explicitly stated otherwise, in connection with functional (1) we consider equation (3) on the interval $[0, N-1]$, i.e. the solution $z$ of (3) is defined on $[0, N+1]$. Note that if we compare the coefficients from the functional and the coefficients from the equation, both $R_{N+1}$ and $C_{N}$ are present in the functional, but neither of them appears in (3) on [0,N-1]. However, for every $m \leq N$ both $R_{N+1}$ and $C_{N}$ appear in the definition of coupled point in the quantity $d_{m}$. In some cases, it is desirable to extend the solution to the interval $[0, N]$, see Remark 2.1 hereafter.

\section{Preliminary results}

In the article, we show that the coupled intervals are convenient for characterization of nonnegativity or positivity of the functional (1). Since the functional (1) contains also a functional with zero boundary conditions on both ends as a subset, the nonnegativity (positivity) of (1) is broken as soon as the nonnegativity (positivity) of the functional with zero boundary conditions is broken. Thus, it is natural to expect that an existence of a conjugate interval implies the existence of a coupled interval. This fact is proved in the following lemma.

Lemma 2.1 Consider equation (3) on $[0, N-1]$ and the corresponding functional (1). Let $m \leq N$. If the interval $(m, m+1]$ is conjugate to 0 , then $(m, m+1]$ is coupled with 0 .

Proof Denote by $z$ the solution of (3) on [0,m-1] which satisfies $z_{0}=0$ and $r_{0} \Phi\left(z_{1}\right)=1$. Suppose that $(m, m+1]$ is conjugate to 0 , i.e. $z_{m} \neq 0$ and $R_{m} z_{m} z_{m+1} \leq 0$. If $\alpha=0$, then $d_{m}$ reduces to

$$
d_{m}=R_{m} z_{m} \Phi\left(\Delta z_{m}\right)+R_{m}\left|z_{m}\right|^{p}=R_{m}\left|z_{m}\right|^{p}\left[\Phi\left(\frac{z_{m+1}}{z_{m}}-1\right)+1\right] .
$$

Graphing the function $f(x)=\Phi(x-1)+1$ we easily find that $\Phi(x-1)+1 \leq 0$ if and only if $x \leq 0$. Hence, $R_{m} z_{m} z_{m+1} \leq 0$ is equivalent to $d_{m} \leq 0$. If $m=N$ then $(m, m+1]$ is coupled with 0 . To finish the proof in the case $m<N$ we have to show that $z \not \equiv \alpha$ on $[m+1, N+1]$. However, since $\alpha=0$ and the solution $z$ is not trivial, the relation $z_{k}=\alpha$ cannot hold for two consecutive indices $k, k+1$.

A representation of the functional (2) by a certain tridiagonal matrix has been used in [4-6] in the original proof of necessary and sufficient conditions for nonnegativity and positivity of (2). However, we lack this representation for the $p$-degree functional and we have to look for another method. A convenient tool for the study of this functional is the so called Picone-type identity, which has been proved in [1], see also [3, Theorem 8.2.1]. Using this identity we derive the following alternative representation of the functional $J$. Note that this representation relies on the existence of the solution of (3) which satisfies $z_{0}=0$ and $z_{k} \neq 0$ for $k \in 1,2, \ldots, N+1$.

Lemma 2.2 Let $z_{k}$ be a solution of (3) on $[0, N-1]$ and suppose that $z_{0}=0$ and $z_{k} \neq 0$ for $k \in[1, N+1]$. For $k \in[1, N]$ define

$$
G_{k}(x, z)=\frac{z_{k+1}}{z_{k}}\left|\Delta x_{k}\right|^{p}-\frac{z_{k+1} \Phi\left(\Delta z_{k}\right)}{z_{k} \Phi\left(z_{k+1}\right)}\left|x_{k+1}\right|^{p}+\frac{z_{k+1} \Phi\left(\Delta z_{k}\right)}{z_{k} \Phi\left(z_{k}\right)}\left|x_{k}\right|^{p} .
$$


Then $G_{k}(x, z) \geq 0$ and

$$
J(x)=\left|x_{N+1}\right|^{p}\left(R_{N+1}+R_{N} \Phi\left(\frac{\Delta z_{N}}{z_{N+1}}\right)-C_{N}\right)+\sum_{k=1}^{N} \frac{R_{k} z_{k}}{z_{k+1}} G_{k}(x, z) .
$$

Proof The nonnegativity of $G_{k}(x, z)$ follows from [1], see also [3, Theorem 8.2.1]. From $z_{k} \neq 0$ for $k \in[1, N]$ and from $[3$, Theorem 8.2.1] we have

$$
R_{k}\left|\Delta x_{k}\right|^{p}-C_{k}\left|x_{k+1}\right|^{p}=\Delta\left\{\left|x_{k}\right|^{p} R_{k} \frac{\Phi\left(\Delta z_{k}\right)}{\Phi\left(z_{k}\right)}\right\}+\frac{R_{k} z_{k}}{z_{k+1}} G_{k}(x, z)
$$

for $k \in[1, N-1]$. Hence,

$$
\begin{aligned}
J(x)= & \left|x_{N+1}\right|^{p} R_{N+1}+R_{N}\left|\Delta x_{N}\right|^{p}-C_{N}\left|x_{N+1}\right|^{p}+R_{0}\left|\Delta x_{0}\right|^{p}-C_{0}\left|x_{1}\right|^{p} \\
& +\sum_{k=1}^{N-1}\left[R_{k}\left|\Delta x_{k}\right|^{p}-C_{k}\left|x_{k+1}\right|^{p}\right] \\
= & \left|x_{N+1}\right|^{p}\left(R_{N+1}-C_{N}\right)+R_{N}\left|\Delta x_{N}\right|^{p}+R_{0}\left|x_{1}\right|^{p}-C_{0}\left|x_{1}\right|^{p} \\
& +\left|x_{N}\right|^{p} R_{N} \Phi\left(\frac{\Delta z_{N}}{z_{N}}\right)-\left|x_{1}\right|^{p} R_{1} \Phi\left(\frac{\Delta z_{1}}{z_{1}}\right)+\sum_{k=1}^{N-1} \frac{R_{k} z_{k}}{z_{k+1}} G_{k}(x, z) .
\end{aligned}
$$

Since $z$ is a solution of (3) for $k=0$ which satisfies $z_{0}=0$, we have

$$
R_{1} \Phi\left(\frac{\Delta z_{1}}{z_{1}}\right)=R_{0} \Phi\left(\frac{\Delta z_{0}}{z_{1}}\right)-C_{0}=R_{0}-C_{0}
$$

and

$$
J(x)=\left|x_{N+1}\right|^{p}\left(R_{N+1}-C_{N}\right)+\left|\Delta x_{N}\right|^{p} R_{N}+\left|x_{N}\right|^{p} R_{N} \Phi\left(\frac{\Delta z_{N}}{z_{N}}\right)+\sum_{k=1}^{N-1} \frac{R_{k} z_{k}}{z_{k+1}} G_{k}(x, z) .
$$

Using the definition of $G_{k}$ and $z_{N} \neq 0, z_{N+1} \neq 0$ we see that

$$
\begin{aligned}
\frac{R_{N} z_{N}}{z_{N+1}} G_{N}(x, z)= & \frac{R_{N} z_{N}}{z_{N+1}}\left(\frac{z_{N+1}}{z_{N}}\left|\Delta x_{N}\right|^{p}\right. \\
& \left.-\frac{z_{N+1} \Phi\left(\Delta z_{N}\right)}{z_{N} \Phi\left(z_{N+1}\right)}\left|x_{N+1}\right|^{p}+\frac{z_{N+1} \Phi\left(\Delta z_{N}\right)}{z_{N} \Phi\left(z_{N}\right)}\left|x_{N}\right|^{p}\right) \\
= & R_{N}\left|\Delta x_{N}\right|^{p}-R_{N} \Phi\left(\frac{\Delta z_{N}}{z_{N+1}}\right)\left|x_{N+1}\right|^{p}+R_{N} \Phi\left(\frac{\Delta z_{N}}{z_{N}}\right)\left|x_{N}\right|^{p}
\end{aligned}
$$

and (6) follows immediately.

As shown in (6), the functional (1) can be written in the form of a sum of two terms. The second term is nonnegative, since it is a sum of other nonnegative terms. The nonnegativity (positivity) of the first term is tied with the nonnegativity (positivity) of the quantity $d_{N}$ from the definition of the coupled interval, as the following lemma shows. 
Lemma 2.3 Let $z$ be a solution of (3) on $[0, N-1]$ which satisfies $z_{0}=0, R_{0} \Phi\left(z_{1}\right)=1, z_{N} \neq 0$ and $z_{N+1} \neq 0$. The interval $(N, N+1]$ is coupled with 0 if and only if

$$
R_{N+1}+R_{N} \Phi\left(\frac{\Delta z_{N}}{z_{N+1}}\right)-C_{N} \leq 0
$$

The interval $(N, N+1]$ is strictly coupled with 0 if and only if the strict inequality holds in (7).

Proof Let $d_{N}$ be defined by (4) with $m=N$. For $\alpha=z_{N+1}$, we have

$$
\begin{aligned}
d_{N} & =R_{N} z_{N} \Phi\left(\Delta z_{N}\right)+R_{N}\left|\Delta z_{N}\right|^{p}+\left|z_{N+1}\right|^{p}\left(R_{N+1}-C_{N}\right) \\
& =R_{N} \Phi\left(\Delta z_{N}\right) z_{N+1}+\left|z_{N+1}\right|^{p}\left(R_{N+1}-C_{N}\right) \\
& =z_{N+1}\left[R_{N} \Phi\left(\Delta z_{N}\right)+R_{N+1} \Phi\left(z_{N+1}\right)-C_{N} \Phi\left(z_{N+1}\right)\right] \\
& =\left|z_{N+1}\right|^{p}\left[R_{N+1}+R_{N} \Phi\left(\frac{\Delta z_{N}}{z_{N+1}}\right)-C_{N}\right] .
\end{aligned}
$$

Since $(N, N+1]$ is coupled (strictly coupled) with 0 if and only if $d_{N} \leq 0\left(d_{N}<0\right)$, the lemma follows.

Remark 2.1 If we extend the solution $z$ from Lemma 2.3 up to $N$, the condition (7) can be written in the form

$$
R_{N+1}+R_{N+1} \Phi\left(\frac{\Delta z_{N+1}}{z_{N+1}}\right) \leq 0
$$

The second term on the left-hand side is closely related with the so called generalized Riccati difference equation

$$
\Delta w_{k}+C_{k}+w_{k}\left(1-\frac{R_{k}}{\Phi\left(\Phi^{-1}\left(R_{k}\right)+\Phi^{-1}\left(w_{k}\right)\right)}\right)=0 .
$$

Really, if $z_{k}$ is solution of (3), then $w_{k}=R_{k} \Phi\left(\Delta z_{k} / z_{k}\right)$ is solution of (8). Thus, the (non)existence of the coupled interval can be characterized with the Riccati equation. For more details related to the Riccati equation technique see [3, Chapter 8] which summarizes known results up to 2005 and also a series of papers by Došlý and Fišnarová [12-14] for some further recent extensions (modified Riccati technique).

\section{Main results}

In this section, we present a necessary and sufficient condition for nonnegativity and positivity of the functional (1) over the class of admissible functions, i.e. functions with zero left endpoint and free right endpoint.

Theorem 3.1 Functional (1) is nonnegative over the class of admissible functions if and only if there is no interval $(m, m+1] \subseteq(1, N]$ coupled with 0 and the interval $(N, N+1]$ is not strictly coupled with 0. 
Proof Suppose that there is no interval $(m, m+1] \subseteq(1, N]$ coupled with 0 and the interval $(N, N+1]$ is not strictly coupled with 0 . Let $z$ be the solution of (3) which satisfies $z_{0}=0$ and $R_{0} \Phi\left(z_{1}\right)=1$. Then $R_{k} z_{k} z_{k+1}>0$ for all $k \in[1, N]$. Really, if there exists $k \in[1, N]$ such that $R_{k} z_{k} z_{k+1} \leq 0$ then $(k, k+1] \subseteq(1, N+1]$ is coupled with 0 according to Lemma 2.1. By Lemma 2.2, the functional (1) allows representation (6) and the nonnegativity of (1) follows from Lemma 2.3 and from the nonnegativity of the terms $G_{k}$ and $R_{k} z_{k} z_{k+1}$ for $k \in[1, N]$.

To prove the opposite implication, suppose, by contradiction, that $J$ is nonnegative and $(m, m+1] \subseteq(1, N]$ is coupled with 0 . Let $z$ and $\alpha$ be the corresponding solution and number from the definition of coupled interval, i.e. $z_{0}=0, R_{0} \Phi\left(z_{1}\right)=1, z_{m} \neq 0, d_{m} \leq 0$ and $z_{k} \not \equiv \alpha$ on $[m+1, N+1]$ if $m \neq N$. Define an admissible function $x$ as in Remark 1.1. Similarly, as in Remark 1.1 we have $J(x)=d_{m} \leq 0$. Since $J$ is nonnegative, the relation $J(x)=0$ holds and $J$ attains its minimum at $x$. Consequently, $x$ satisfies the corresponding EulerLagrange equation (3) on $[0, N-1]$ and hence $x \equiv z$ on $[0, N+1]$. However, $x_{k} \equiv \alpha$ on $[m+1, N+1]$ and hence also $z_{k} \equiv \alpha$ on $[m+1, N+1]$ which contradicts the definition of the coupled interval. In a similar way, if $J$ is nonnegative and $(N, N+1]$ is strictly coupled, then the function defined in Remark 1.1 satisfies $J(x)=d_{N}<0$ which contradicts nonnegativity of $J$.

Remark 3.1 The sum $\sum_{k=1}^{N} \frac{R_{k} z_{k}}{z_{k+1}} G_{k}(x, z)$ which appears on the right-hand side of (6) equals zero if and only if $x$ is a constant multiple of $z$ on $[0, N+1]$ (see [3] for details). The remaining part from the right hand side can be written in the form

$$
\left|x_{N+1}\right|^{p} R_{N+1}\left(1+\Phi\left(\frac{\Delta z_{N+1}}{z_{N+1}}\right)\right) \text {, }
$$

where $\Delta z_{N+1}$ is evaluated by extending the solution $z$ from the interval $[0, N-1]$ to $[0, N]$. Now it is easy to see that this expression equals zero if and only if $x_{N+1}=0$ or $z_{N+2}=0$. These conditions together characterize the case when a nonnegative functional becomes zero.

The following theorem presents a necessary and sufficient condition for the positivity of (1). Note that the difference between positivity and nonnegativity is only at $(N, N+1]$. The interval $(N, N+1]$ is allowed to be coupled with 0 but not strictly coupled in Theorem 3.1, i.e. $d_{N} \geq 0$. This condition is sharpened and the possibility $d_{N}=0$ is excluded in a necessary and sufficient condition for positivity of (1).

Theorem 3.2 Functional (1) is positive over the class of admissible functions if and only if there is no interval $(m, m+1] \subseteq(1, N+1]$ coupled with 0 .

Proof If there is no interval $(m, m+1] \subseteq(1, N+1]$ coupled with 0 , then $(N, N+1]$ is not strictly coupled and the functional (1) is nonnegative by Theorem 3.1. To prove the positivity we have to show that $J(x)$ equals zero if and only if $x$ equals zero on $[0, N+1]$. By Lemma 2.1, there is no interval $(m, m+1] \subseteq(1, N+1]$ conjugate with 0 and hence a solution which satisfies $z_{0}=0, R_{0} \Phi\left(z_{1}\right)=1$ satisfies $R_{k} z_{k} z_{k+1}>0$ for $k \in[1, N]$. By Lemma 2.2, the functional $J(x)$ possesses representation in the form (6). This representation consists of two nonnegative terms (see also Lemma 2.3) and to finish the proof we have to show that both terms equal zero if and only if $x$ equals zero on $[0, N+1]$. Since $z_{k} \neq 0$ on $[0, N+1]$, 
the second term in (6) vanishes only if all the terms $G_{k}(x, z)$ vanish on $[1, N]$ which means that $x$ is a constant multiple of $z$ on $[0, N+1]$ (see [3, Theorem 8.2.1]). Since $(N, N+1]$ is not coupled with 0 , by Lemma 2.3 we have

$$
R_{N+1}+R_{N} \Phi\left(\frac{\Delta z_{N}}{z_{N+1}}\right)-C_{N}>0
$$

Hence, $J(x)=0$ implies $x_{N+1}=0$. Since $x$ is a constant multiple of $z$ and $z_{N+1} \neq 0$, we have $x_{k}=0$ for all $k \in[0, N+1]$ and the functional is positive definite.

To prove the other implication, suppose that the functional (1) is positive. Then it is also nonnegative and by Theorem 3.1 there is no interval $(m, m+1] \subseteq(1, N]$ coupled with 0 and $(N, N+1]$ is not strictly coupled with 0 . To finish the proof it is sufficient to prove that $(N, N+1]$ is not coupled. However, if $z$ is a solution which satisfies $z_{0}=0, R_{0} \Phi\left(z_{1}\right)=1$, and for some $\alpha$ we have $d_{N}=0$, then the function $x$ defined by (5) is nontrivial and $J(x)=d_{N}=$ 0 , which contradicts positivity of $J$.

Remark 3.2 If we consider linear case $p=2$ in Theorems 3.1, 3.2 and the scalar case with zero boundary condition at the left endpoint in [4, Theorems 5.3, 5.4], then the corresponding statements, i.e. the equivalence between the nonnegativity of the functional and nonexistence of the coupled point, coincide.

\section{Competing interests}

The author declares that he has no competing interests.

\section{Acknowledgement}

The research was supported by the Grant P201/10/1032 of the Czech Science Foundation.

Received: 16 March 2012 Accepted: 21 June 2012 Published: 3 July 2012

\section{References}

1. Řehák, P: Oscillatory properties of second order half-linear difference equations. Czechoslov. Math. J. 51(126), 303-321 (2001)

2. Mařík, R: Comparison theorems for half-linear second order difference equations. Arch. Math. 36, 513-518 (2000)

3. Došlý, O, Řehák, P: Half-Linear Differential Equations. North-Holland Mathematics Studies, vol. 202. Elsevier, Amsterdam (2005)

4. Hilscher, R, Zeidan, V: Nonnegativity and positivity of quadratic functionals in discrete calculus of variations: survey. J. Differ. Equ. Appl. 11(9), 857-875 (2005)

5. Hilscher, R, Zeidan, V: Coupled intervals in the discrete optimal control. J. Differ. Equ. Appl. 10(2), 151-186 (2004)

6. Hilscher, R, Zeidan, V: Coupled intervals in the discrete calculus of variations: necessity and sufficiency. J. Math. Anal. Appl. 276, 396-421 (2002)

7. Došlá, Z, Došlý, O: Quadratic functionals with general boundary conditions. Appl. Math. Optim. 36, $243-262$ (1997)

8. Došlá, Z, Zezza, P: Conjugate points in the theory of variations and optimal control theory via the quadratic form theory. Differ. Equ. Dyn. Syst. 2, 137-152 (1994)

9. Zeidan, V, Zezza, P: Variable end points in the calculus of variations: coupled points. In: Bensoussan, A, Lions, JL (eds.) Lecture Notes in Control and Information Sciences, vol. 111, pp. 372-380. Springer, Berlin (1988)

10. Zeidan, V Z Zezza, P: Coupled points in the calculus of variations and applications to periodic solutions. Trans. Am. Math. Soc. 315(1), 323-335 (1989)

11. Mařík, R: A half-linear differential equation and corresponding variational problem. Nonlinear Anal. 45, 203-211 (2001)

12. Došlý, O, Fišnarová, S: Perturbation principle in discrete half-linear oscillation theory. Stud. Univ. Žilina Math. Ser. 23(1), 19-28 (2009)

13. Došlý, O, Fišnarová, S: Linearized Riccati technique and (non-)oscillation criteria for half-linear difference equations. Adv. Differ. Equ. 2008, 1-18 (2008)

14. Došlý, O, Fišnarová, S: Summation characterization of the recessive solution for half-linear difference equations. Adv. Differ. Equ. 2009, 1-16 (2009) 